\title{
Sistema de control digital de bajo coste de un motor de corriente continua para usos didácticos
}

\author{
G. Olivares ${ }^{1}$, A. Olivares ${ }^{2}$, F. Gómez ${ }^{1}$, M. Damas ${ }^{1}$. \\ 1) Departamento de Arquitectura y Tecnología de Computadores. ETSI Informática y de \\ Telecomunicación. Universidad de Granada \\ \{gonzalo, aolivares, frgomez, mdamas\}@ugr.es \\ 2) Nazaríes Information Technologies S.L. (http://www.nazaries.com) \\ alberto.olivares@nazaries.com
}

\begin{abstract}
Resumen. Este trabajo describe el desarrollo de un conjunto de experiencias prácticas de diseño e implementación de algunas técnicas de simulación y control digital de un motor de corriente continua llevadas a cabo con elementos físicos de muy bajo coste. El sistema de control desarrollado se está empleando en las prácticas de las asignaturas impartidas por el Área de Ingeniería de Sistemas y Automática y el Departamento de Arquitectura y Tecnología de Computadores de la Universidad de Granada.
\end{abstract}

Palabras Claves: Control digital, Motor de corriente continua, Mecatrónica, Bajo Coste, Docencia Experimental.

\begin{abstract}
This work describes the development of a set of practical experiences in the design and implementation of various simulation and digital control techniques of a DC motor carried out with very low cost elements. The control system developed is being used in the practices of the subjects taught by the Systems Engineering and Automation Area and the Department of Architecture and Computer Technology of the University of Granada.
\end{abstract}

Keywords: Digital-Control, DC motor, Mechatronics, Low-cost, Experimental Learning.

\section{Introducción}

El motor de corriente continua es uno de los principales componentes que se utilizan tanto en Robótica como en Mecatrónica. Conocer bien su comportamiento, modelado y métodos de control es muy importante para avanzar y abordar más adelante el control de sistemas mecánicos más complejos. Además, controlar la posición angular del eje de un motor (servo-motor) o la velocidad angular del mismo, permite de una forma sencilla, experimentar y asimilar correctamente un conjunto amplio de conceptos relacionados con el desarrollo de sistemas discretos de control, tales como: funciones de transferencia continuas y discretas, estabilidad, controlabilidad, observabilidad, controladores PID, control LQR, representación en el espacio de 
estados, control por ubicación de polos, controladores-observadores, o bien simplemente experimentar con filtros digitales, análisis de ruido, etc. Existen muchos equipos de prácticas con motores de corriente continua, que se incluyen en los catálogos de diversos fabricantes [1][2]. En la mayoría de los casos, su coste es elevado, y con bastante frecuencia, el alumno tiene que aprender herramientas específicas de software propietario del fabricante. A veces se utiliza una nomenclatura distinta a la que el alumno ha aprendido en las clases teóricas, o bien se hace hincapié en conceptos que no siempre son esenciales. Por tanto, lo que la mayoría de las veces se dispone en el laboratorio son sistemas didácticos cerrados, que no permiten que el alumno experimente fácilmente, de forma autónoma y a bajo coste, lo que en las clases teóricas ha aprendido. Por supuesto, ello dificulta que el alumno se pueda llevar el material incluso a casa para experimentar con más tranquilidad y ensayar por si mismo las soluciones propuestas en clase de teoría. Por tanto, pretendemos diseñar un pequeño equipo, muy barato, fácil de transportar y de reparar, con un conjunto básico de guiones de prácticas, y que a partir de ahí el alumno experimente después por sí sólo, y use posteriormente estas técnicas en maquetas electromecánicas más complejas que integren motores DC. En este trabajo, a continuación se describe el hardware que se propone usar,

\section{Descripción del hardware}

Dado que lo que pretendemos es realizar un sistema de control digital para la regulación de la posición y también la velocidad angular de un motor de corriente continua, proponemos utilizar un motor de baja potencia y bajo coste que incorpore además un codificador incremental. Hemos seleccionado el motor Namiki 22CL3501PG de 12 VDC, con una relación de engranajes de 80:1 [3]. El codificador dispone de dos opto-acopladores, tal y como puede apreciarse en la figura 1, en la que además se muestra una fotografía del motor. Permite medir la posición angular (en los dos sentidos), con 640 pulsos por vuelta, lo que equivale a una resolución de 0.0098175 radianes $(0.56$ grados).
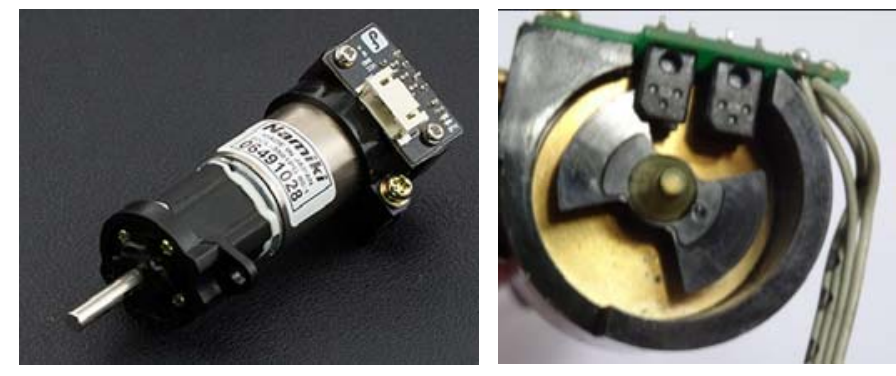

Fig 1. Motor y detalle del codificador incremental

Como controlador se utiliza una tarjeta Arduino Mega 2560 R3 [4]. Podríamos haber utilizado alguna otra tarjeta Arduino de menor coste, sin embargo esta es más apropiada para poder utilizarse en tiempo real con el modo External de tiempo real de Simulink [4]. Para suministrar la potencia adecuada, utilizaremos la tarjeta Arduino 
Motor Shield [5], que incorpora el circuito integrado L293 (Puente H dual), mediante el cual se pueden controlar la velocidad angular de hasta dos motores de corriente continua, mediante dos salidas PWM. Admite un consumo de corriente de hasta 2 amperios. En la figura 2 se muestra una fotografía del sistema completo.

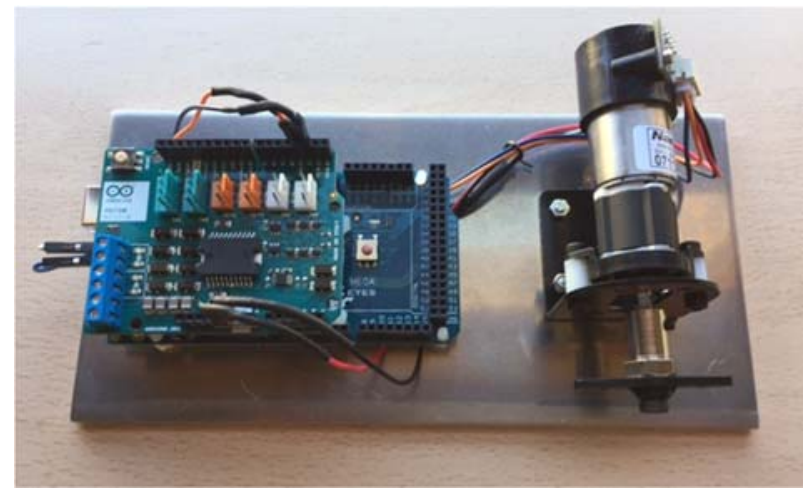

Fig 2. Fotografía del sistema completo

\section{Objetivos de aprendizaje}

Presentamos a continuación una lista de tareas experimentales desarrolladas con el equipo anteriormente descrito:

1. Obtención experimental de parámetros del motor.

2. Funciones de transferencia continua y discreta: cálculo teórico y experimental.

3. Representación en el espacio de estados continuo y discreto del modelo del motor DC.

4. Diseño y simulación de Control PID discreto de posición y de velocidad angular.

5. Estudio del codificador incremental del motor y desarrollo de software en $\mathrm{C}++\mathrm{y}$ Simulink para la medida de ángulo y velocidad angular.

6. Control de la velocidad del motor en lazo abierto.

7. Implementación en $\mathrm{C}++\mathrm{y}$ Simulink de controlador PID discreto.

8. Diseño y programación de control de posición y velocidad angular por realimentación en el espacio de estados y ubicación de polos.

9. Control por realimentación en el espacio de estados, con integrador.

10. Control LQR.

11. Diseño, simulación e implementación en tiempo real de controladorobservador

12. Modelado y simulación del sistema con SolidWorks y Sinmechanics.

Todas estas unidades didácticas han sido planificadas y ensayadas con éxito. 


\section{Resultados obtenidos}

A continuación se describen algunos de los módulos experimentales y los resultados obtenidos.

\subsection{Control del motor en lazo abierto.}

En la figura 3 se presenta un módulo realizado con Simulink para el control de velocidad del motor. Se puede seleccionar el sentido de giro mediante la activación de un pin digital; asimismo, la velocidad se regula con una salida PWM.

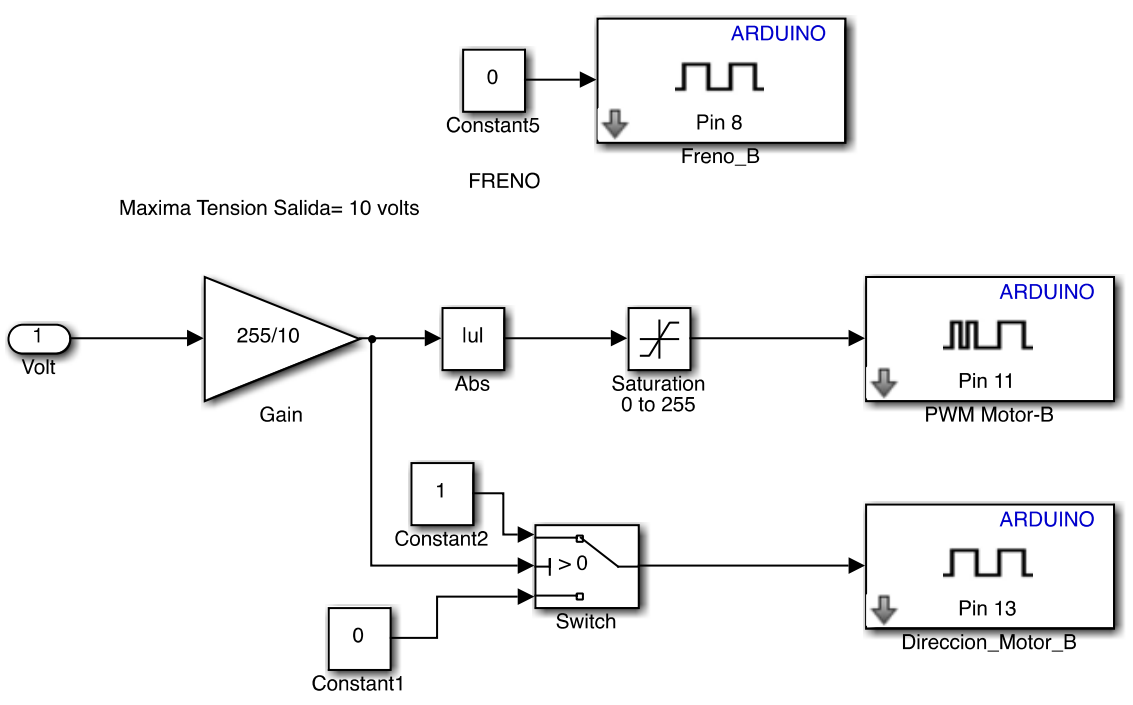

Fig 3. Control de la velocidad y sentido de giro del motor en lazo abierto

\subsection{Control PID}

Los parámetros del controlador PID (constantes proporcional, diferencial e integral) se diseñan a partir de la función de transferencia del motor y en función de la respuesta deseada (sobrepasamiento máximo y tiempo de asentamiento). Se han utilizado los siguientes procedimientos:

- Diseño por ubicación de los polos de la respuesta final deseada.

- Método de Ziegler Nichols.

- Procedimiento de auto-tuning de Matlab y de Simulink con simulación previa de resultados. 
Una vez diseñado el controlador PID, este se implementó de nuevo con Simulink, visualizando en tiempo real la respuesta ante un cambio de consigna periódico (ver figura 4).

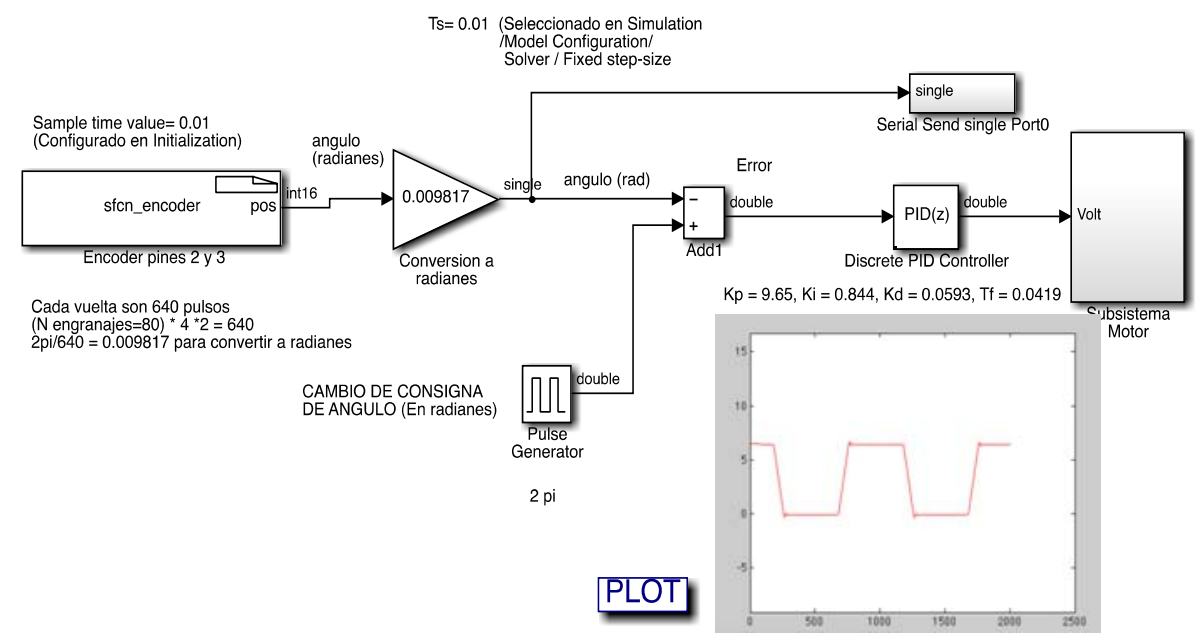

Fig 4. Control PID de posición angular realizado con Simulink y respuesta obtenida

\subsection{Control LQR}

A partir de las ecuaciones diferenciales que definen el comportamiento físico de un motor de corriente continua:

$$
\begin{aligned}
& K i=J \ddot{\theta}+b \dot{\theta} \Rightarrow \ddot{\theta}=-\frac{b}{J} \dot{\theta}+\frac{K}{J} \mathrm{i} \\
& V-K \dot{\theta}=R \dot{R}+L \dot{i} \quad \Rightarrow \quad \dot{i}=-\frac{K}{L} \dot{\theta}-\frac{R}{L} i+\frac{V}{L}
\end{aligned}
$$

donde i es la corriente del motor, $\mathrm{J}$ es el momento de inercia del eje, $\mathrm{K}$ la constante de fuerza electromotriz, con el mismo valor que la constante de par motor $(\mathrm{K}=\mathrm{Kt}=\mathrm{Ke}), \mathrm{R}$ la resistencia, $\mathrm{L}$ la autoinducción, $\mathrm{b}$ el coeficiente de fricción, $\mathrm{y} \mathrm{V}$ la entrada de control (voltaje aplicado al motor), se obtiene el modelo continuo del motor en el espacio de estados: 


$$
\left(\begin{array}{c}
\dot{\theta} \\
\ddot{\theta} \\
\dot{i}
\end{array}\right)=\left(\begin{array}{ccc}
0 & 1 & 0 \\
0 & -b / J & K / J \\
0 & -K / L & -R / L
\end{array}\right)\left(\begin{array}{c}
\theta \\
\dot{\theta} \\
i
\end{array}\right)+\left(\begin{array}{c}
0 \\
0 \\
1 / L
\end{array}\right) V
$$

y también la funciones de transferencia con respecto a la posición angular:

$$
\frac{\theta(s)}{V(s)}=\frac{K}{s\left[J L s^{2}+(R J+L b) s+\left(R b+K^{2}\right)\right]}
$$

y la función de transferencia con respecto a la velocidad angular:

$$
\frac{\omega(s)}{V(s)}=\frac{K}{J L s^{2}+(R J+L b) s+\left(R b+K^{2}\right)}
$$

Con la representación en el espacio de estados, hemos implementado un sistema de control de posición angular por realimentación en el espacio de estados (ver figura 5), donde las constantes de realimentación se calculan mediante un Regulador Cuadrático Lineal (LQR) discreto que optimiza la respuesta de control, minimizando además el coste energético [7]. Se trata de obtener las constantes $\mathrm{K}$ de realimentación en el espacio de estados discreto, que minimizan la función de coste:

$$
J=\sum_{k=0}^{\infty}\left[x_{k}^{T} Q x_{k}+u_{k}^{T} R u_{k}\right]
$$

Para ello se realiza la elección apropiada de los parámetros de peso Q, R, utilizando la regla de Bryson. Se ha discretizado el modelo, con un periodo de muestreo de 0.01 segundos. En la figura 6, se puede apreciar que las respuestas simulada y real se aproximan muy bien; sin embargo, hemos podido comprobar que la medida de la corriente eléctrica del motor, no se realiza con precisión con la tarjeta Arduino Shield Motor, a pesar de que dispone de una salida analógica para ello. 


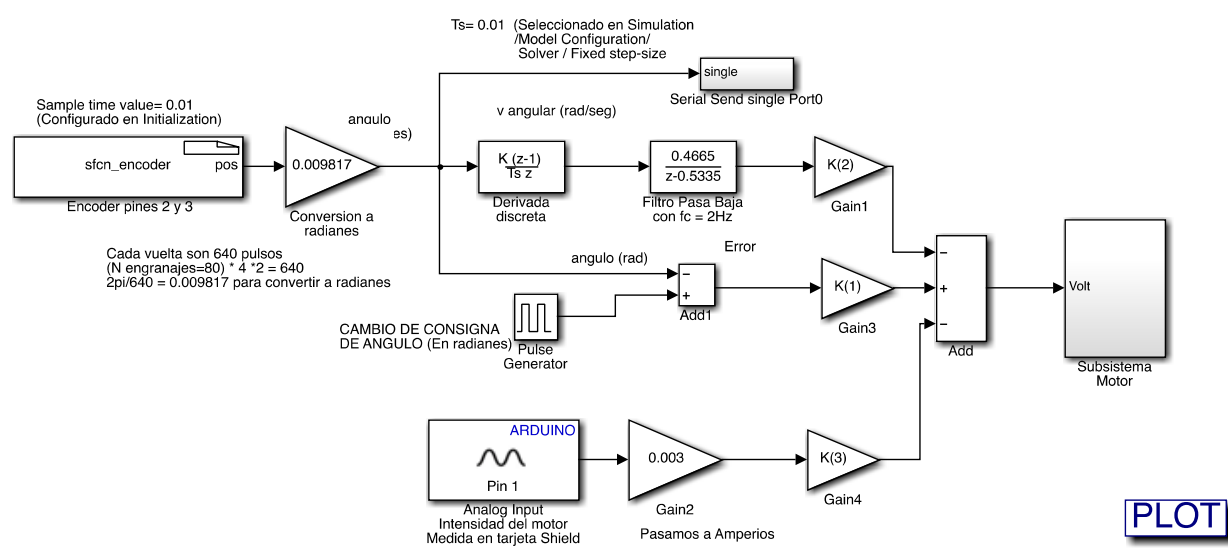

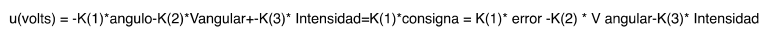

K se obtiene con IarA,B, , R desde Workspace
usando SimulaServoNamikiSimulink.m

Fig 5. Diseño del control LQR del servo motor, utilizando la biblioteca de Arduino para Simulink.
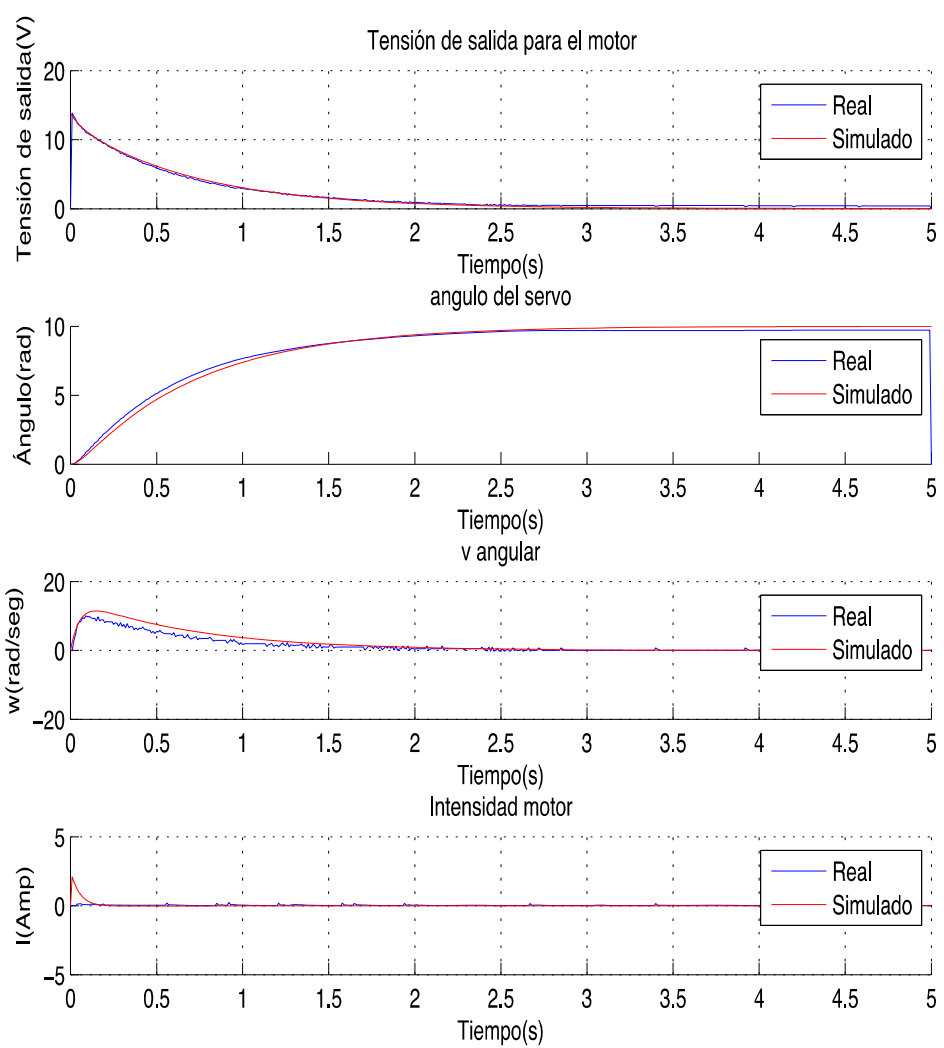

Fig 6. Comparación de resultados del sistema de control simulado con el real. 


\subsection{Controlador-Observador}

Cuando no puede medirse con precisión alguna de las variables de estado, se puede utilizar un observador de estados discreto (siempre que el sistema sea observable) [6] a partir del modelo de estados y de la salida, que en nuestro caso es la posición angular del eje del motor. El diseño de las constantes L de realimentación del observador y las constantes $\mathrm{K}$ de realimentación del controlador, se realiza previamente mediante Matlab. Los resultados obtenidos en tiempo real con la maqueta del motor son también satisfactorios y nos permiten mostrar fácilmente al alumno la utilidad del diseño de sistemas de control con observadores.

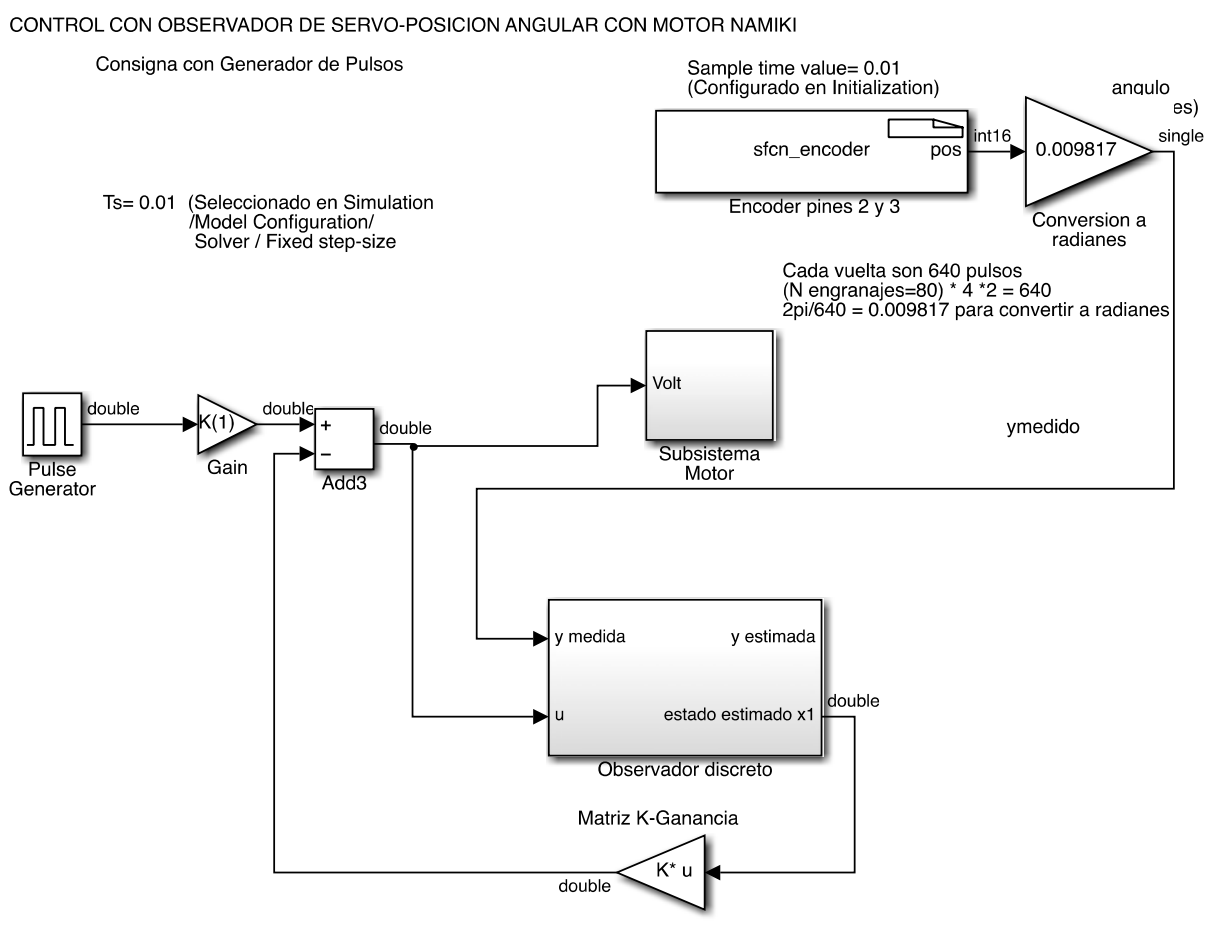

$K$ y $L$ del observador se obrienen ejcutando SimulaservoNamikiObservador

Fig 7. Controlador-Observador 


\section{Conclusiones}

Se ha diseñado un sistema y un conjunto de experiencias prácticas para el estudio de las principales técnicas de control digital de la posición y velocidad de un motor de corriente continua. El sistema es de bajo coste, fácil de programar mediante Simulink o en lenguaje $\mathrm{C}$ de Arduino), y sencillo de mantener en un laboratorio de prácticas. Hemos podido ensayar (simular y controlar físicamente en tiempo real), varias configuraciones de control de posición y velocidad angular, tales como:

- Diseño control discreto PID a partir de la f.d.t o bien con el método de sintonización de Ziegler-Nichols.

- A partir del modelo en espacio de estados, se diseña: a) un sistema de control por realimentación de estados y ubicación de polos, b) Control LQR (Regulador Cuadrático Lineal) y c) un controlador-observador.

La programación gráfica de Simulink en tiempo real es sencilla y muy intuitiva. Dado que disponemos del modelo matemático del motor (función de transferencia discreta y su representación en el espacio de estados), se puede comparar la respuesta del sistema simulado con la respuesta real del sistema de control digital de manera muy rápida. El bajo coste de los materiales utilizados permite la fabricación a mayor escala de las maquetas de prácticas implementadas, por lo que, sin duda, a corto plazo los alumnos podrán disponer del material adecuado para simultanear las prácticas con la teoría o incluso para trabajar en casa.

Agradecimientos. Al Secretariado de Innovación Docente de la Universidad de Granada por los Proyectos de Innovación Docente concedidos que nos han permitido financiar la adquisición de algunas de las maquetas de laboratorio utilizadas en prácticas.

\section{Referencias}

1 Alecoop: http://www.alecop.com/equipamiento-didactico/areas/regulacion-electronica-demotores/. Accedido el 12/07/2017.

2. Feedback-instruments. http://www.feedback-instruments.com/. Accedido el 12/07/2017

3. Dfrobot. https://www.dfrobot.com/product-1460.html. Accedido el 12/07/2017

4. Arduino support from Simulink. http://es.mathworks.com/hardware-support/arduinosimulink.html. Accedido el 12/07/2017.

5. Arduino. https://www.arduino.cc. Accedido el 12/07/2017.

6. Ogata, N. "Ingeniería de Control Moderna". ISBN: 9788483226605

7. Bishop. R.H: "Sistemas de Control Moderno". ISBN-10: 8420544019. 2005. 